\title{
Phenotypic Divergence for Yield and Drought Tolerance Related Traits in Groundnut Genotypes under Rainfed Conditions
}

\author{
G. Kavitha* and M. Reddi Sekhar \\ Department of Genetics \& Plant Breeding, S.V. Agricultural College, ANGRAU, \\ Tirupathi, Andhra Pradesh-517 502, India \\ *Corresponding author
}

Keywords

Groundnut,

Phenotypic

diversity, Cluster analysis, Pod yield

Article Info

Accepted:

24 February 2018

Available Online:

10 March 2018

\section{A B S T R A C T}

Fifty groundnut genotypes were evaluated in kharif 2014 under rainfed conditions. Data was collected on sixteen yield and drought related traits to assess the phenotypic diversity and to investigate the relationship between pod yield and other drought tolerance related traits in groundnut. Coefficient of variation ranged from 0.72 to 13.33 . Clustering based on groundnut genotypes separated the measured traits into three main groups and based on traits it separated groundnut genotypes into five major groups. Number of pods per plant, dry matter per plant, SLA at 80 DAS, number of sound mature kernels per plant, kernel yield per plant and SLA at 60 DAS were the most related traits with pod yield per plant. The genotypes MLTG(SB)-3, MLTG(SB)-6, MLTG(VB)-11 and MLTG(VB)-2 could be utilized for improving pod yield per plant and its component traits.

\section{Introduction}

Groundnut is one of the main oilseed and food legume crop of India. Drought is the most important factor limiting the yield potential of the genotypes under rainfed conditions.

Crop physiologists have identified number of traits that would help the breeder in development and identification of moisture stress tolerant genotypes with high yield potential. Development of high yielding pure line cultivars coupled with water use efficient traits is the major breeding objective of groundnut genetic improvement in order to obtain high productivity under rainfed conditions.

Cluster analysis could be used as a statistical tool to bring information about appropriate cause and effect relationship between yield and yield components. This technique of using Euclidean distance for clustering the genotypes and traits were validated already in mungbean by Basnet et al., (2014) based on quantitative parameters, Katiyar et al., (2009) and Singh et al., (2010) in Brassica it was validated by Binodh et al., (2013) and in 
Pigeon pea it was validated by Yogendra et al., (2013). Pod yield is the primary factor affecting the economical value in groundnut and breeding efforts in increasing pod yield are being conducted. For effective selection, information on nature and magnitude of variation in plant materials, association of different traits with pod and among themselves is necessary. Sixteen yield and drought tolerance traits were taken in this investigation to assess the distinctiveness and the level of phenotypic variation. The paper deals with identification of genotypes as possible sources of parental materials and also identification of traits which may be useful in breeding higher-yielding genotypes with drought tolerance related traits.

\section{Materials and Methods}

The material for the present study comprised of 50 groundnut genotypes, grown in a Randomized block design with three replications at Sri Venkateswara Agricultural College dry land farm Tirupati during kharif, 2014. Each treatment was sown in one row of $3 \mathrm{~m}$ length by adopting a spacing of $30 \mathrm{X} 10$ $\mathrm{cm}$. observations were recorded on randomly chosen ten competitive plants for all characters viz., number of primary branches per plant, number of pods per plant, number of seeds per pod, number sound mature kernels per plant, dry matter per plant (g), pod yield per plant $(\mathrm{g})$, kernel yield per plant $(\mathrm{g})$, shelling per cent, SLA at 60 DAS, SLA at 80 DAS, SCMR at 60 DAS, SCMR at 80 DAS, leaf nitrogen $(\%)$ content at 60 DAS and leaf nitrogen $(\%)$ content at $80 \mathrm{DAS}$.

The characters viz., days to $50 \%$ flowering and days to maturity were recorded on per plot basis. Leaf nitrogen (\%) content values were transformed using arc-sine transformation. Analysis of variance was carried out as per the method suggested by Panse and Sukhatme (1961). Genetics components of variance were obtained as outlined by Johnson et al., (1956). Cluster analysis was used to arrange a set of variables (genotypes and traits) into clusters. Its objective was to sort variables into groups, so the magnitude of association was strong between members of the same cluster and weak between members of different clusters. Each cluster described the class to which its members belonged and this description may be abstracted through use of the particular to the general class or type. The cluster analysis was performed using a measure of similarity levels and Euclidean distance (Eisen et al., 1998) using Minitab version 14 package.

\section{Results and Discussion}

The analysis of variance for 16 characters in 50 genotypes revealed that the genotypes differed significantly for all the characters indicating the existence of sufficient variability in the material studied. Coefficient of variation ranged from 0.72 to 13.33 . Clustering based on groundnut genotypes separated the measured traits into three main groups under rainfed conditions (Figure 1). There were days to fifty percent flowering, days to maturity, shelling percentage and number of kernels per pod in one cluster, number of pods per pod, dry matter per plant, SLA at 80 DAS, number of sound mature kernels per plant, pod yield per plant, kernel yield per plant and SLA at 60 DAS in the second cluster and likewise number of primary branches per plant, SCMR at 60 DAS, leaf nitrogen content at $60 \mathrm{DAS}, \mathrm{SCMR}$ at $80 \mathrm{DAS}$ and leaf nitrogen content at 80 DAS. Therefore, it seems that number of pods per plant, dry matter per plant, SLA at 80 DAS, number of sound mature kernels per plant, kernel yield per plant and SLA at 60 DAS were the most related traits with pod yield per plant while some other traits like kernel yield per plant, SLA at 60 DAS and number of primary branches per plant were grouped in other clusters. 
Fig.1 Similarity levels of the estimated traits in 50 groundnut genotypes using the hierarchical cluster analysis under rainfed conditions

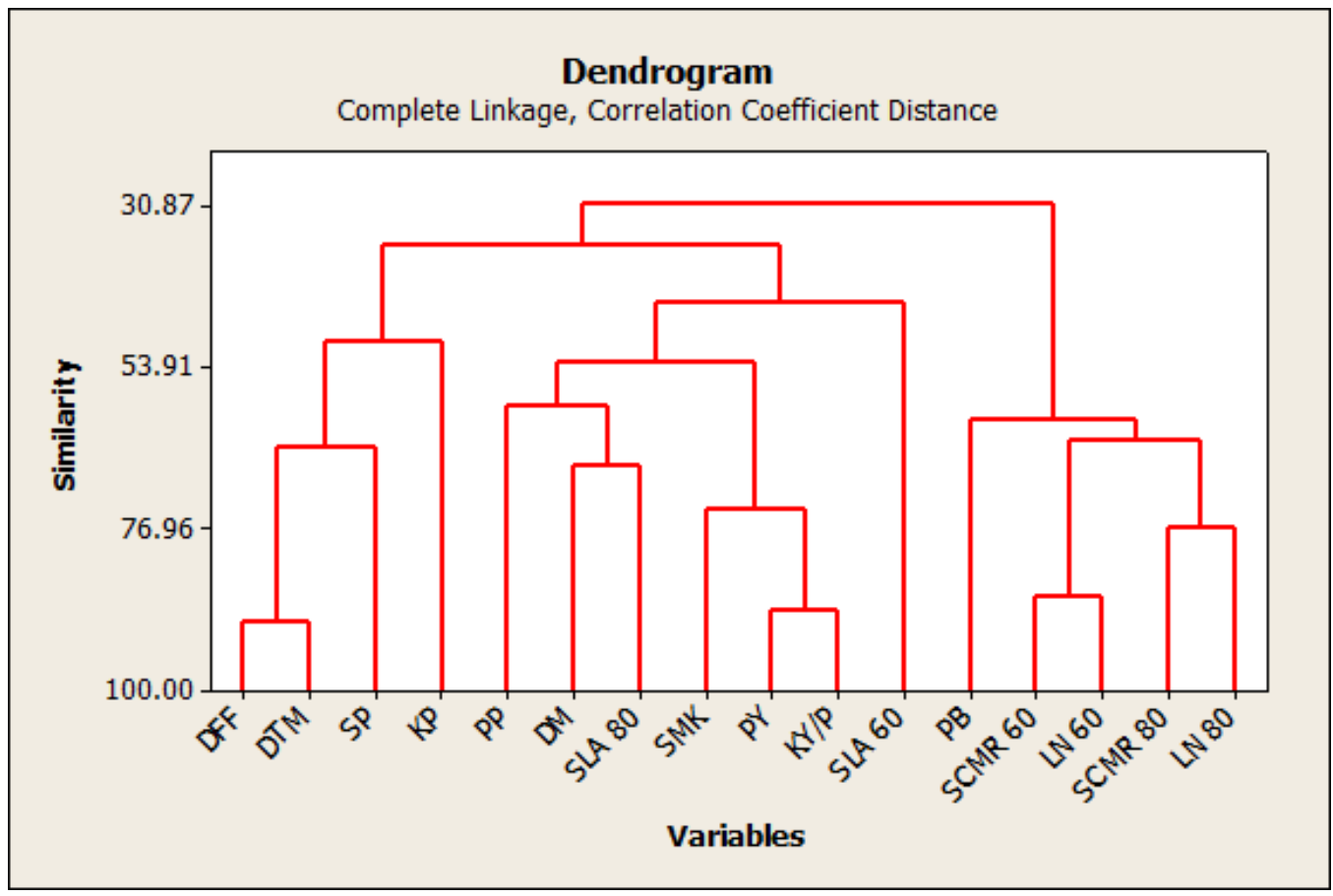

Fig.2 Similarity levels of the estimated fifty groundnut genotypes using the hierarchical cluster analysis under rainfed conditions

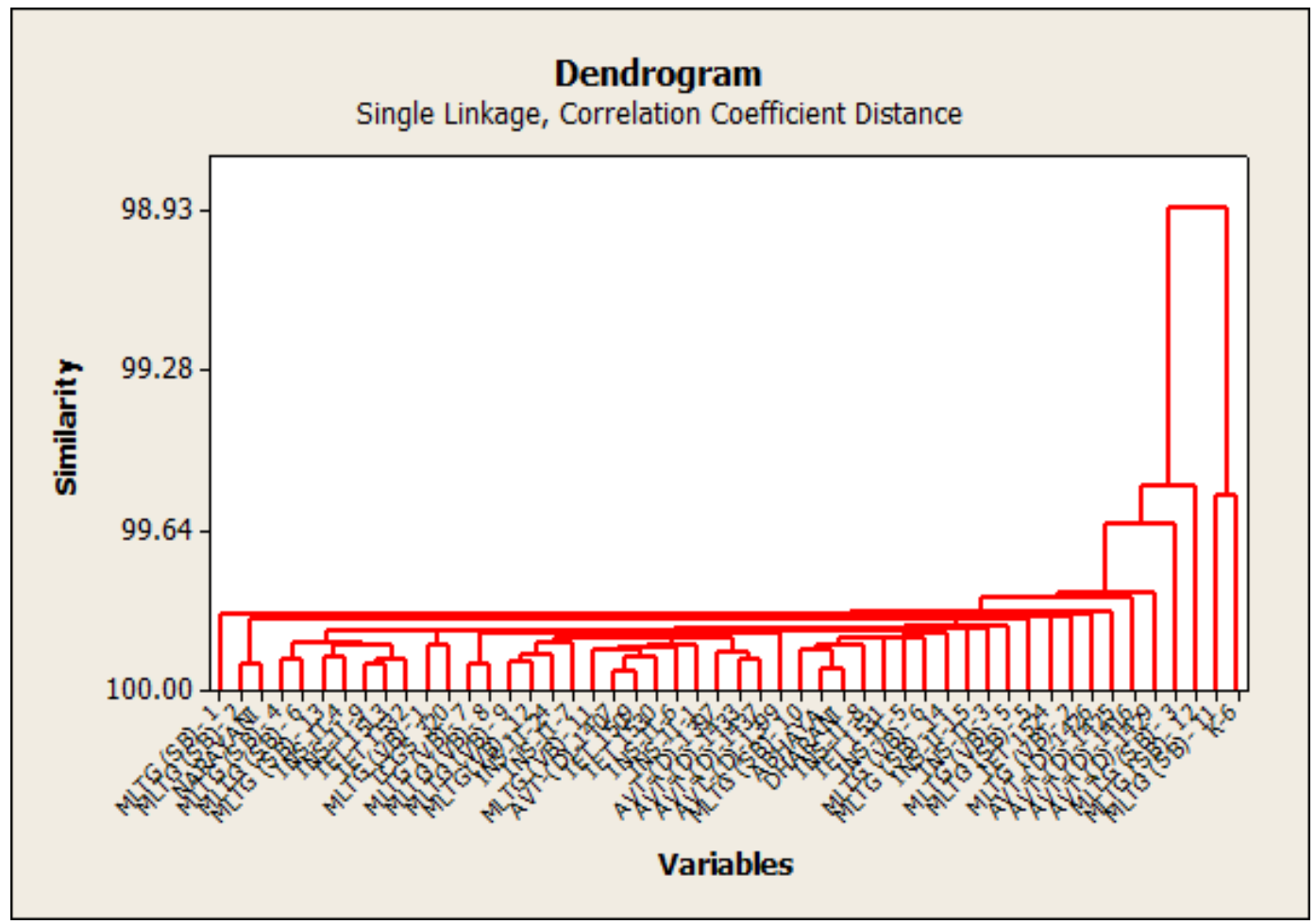


Table.1 Mean performance of fifty groundnut genotypes for sixteen pod yield and drought tolerance related traits in groundnut

\begin{tabular}{|c|c|c|c|c|c|c|c|c|c|c|c|c|c|c|c|c|c|}
\hline $\begin{array}{l}\text { Sl. } \\
\text { No. }\end{array}$ & Genotype & DFF & DTM & PB & PP & KP & SMK & $\begin{array}{l}\text { DM } \\
(\mathrm{g})\end{array}$ & $\begin{array}{l}\text { PY } \\
(\mathrm{g})\end{array}$ & $\begin{array}{l}\text { KY/ } \\
\mathbf{P}(\mathbf{g})\end{array}$ & $\begin{array}{l}\text { SP } \\
(\%)\end{array}$ & $\begin{array}{l}\text { SLA } 60 \\
\mathrm{Cm}^{2} \mathrm{~g}^{-1}\end{array}$ & $\begin{array}{l}\text { SLA 80 } \\
\mathrm{Cm}^{2} \mathrm{~g}^{-1}\end{array}$ & $\begin{array}{l}\text { SCMR } \\
60\end{array}$ & $\begin{array}{l}\text { SCMR } \\
80\end{array}$ & $\begin{array}{l}\text { LN } 60 \\
(\%)\end{array}$ & $\begin{array}{l}\text { LN } 80 \\
(\%)\end{array}$ \\
\hline 1 & $\operatorname{MLTG}(\mathrm{SB})-1$ & 27.67 & 98 & 4.33 & 19 & 1.77 & 20.33 & 31 & 12 & 7.17 & 58.42 & 147.78 & 133.46 & 34.86 & 37.5 & 3.19 & 3.38 \\
\hline 2 & MLTG (SB)- 2 & 26 & 91.67 & 5 & 16 & 1.85 & 18.33 & 24 & 20.6 & 11.42 & 55.43 & 154.27 & 149.47 & 37.67 & 36.21 & 3.52 & 3.33 \\
\hline 3 & MLTG (SB)- 3 & 25.67 & 94.67 & 7.67 & 17.67 & 1.92 & 19 & 20.93 & 25.13 & 11.13 & 44.3 & 137.29 & 154.83 & 43.57 & 42.73 & 3.82 & 3.54 \\
\hline 4 & MLTG (SB)- 4 & 27.67 & 99 & 4 & 15.67 & 1.78 & 21.67 & 13.73 & 18.6 & 9.38 & 50.89 & 146.77 & 133.35 & 39.67 & 41.67 & 3.54 & 3.49 \\
\hline 5 & MLTG (SB)- 5 & 25.67 & 93 & 6.67 & 14.33 & 1.88 & 19 & 14 & 17.28 & 12.76 & 74.23 & 155.53 & 123.56 & 46.26 & 37.8 & 3.87 & 3.29 \\
\hline 6 & MLTG (SB)- 6 & 26.67 & 95.67 & 7.33 & 18.33 & 1.8 & 23.67 & 21.5 & 23.53 & 11.46 & 50.76 & 145.08 & 134.87 & 39.12 & 42.55 & 3.66 & 3.54 \\
\hline 7 & $\operatorname{MLTG}(\mathrm{SB})-10$ & 28.67 & 100 & 6.67 & 20.33 & 1.75 & 16 & 15.17 & 17.17 & 12.99 & 75.67 & 150.09 & 139.25 & 41.85 & 43.29 & 3.6 & 3.54 \\
\hline 8 & MLTG (SB)- 11 & 29 & 99 & 6 & 15.67 & 1.78 & 12.67 & 19.83 & 13.6 & 7.35 & 50.47 & 217.41 & 143.45 & 38.89 & 39.74 & 3.58 & 3.48 \\
\hline 9 & MLTG (SB)- 12 & 26.33 & 95 & 5 & 21 & 1.79 & 17.67 & 18.33 & 11.9 & 8.83 & 74.21 & 152.93 & 195.04 & 45.63 & 45.87 & 3.87 & 3.71 \\
\hline 10 & MLTG (SB)- 13 & 27.33 & 97 & 6.33 & 18.33 & 1.69 & 18.67 & 19 & 13.67 & 7.22 & 56.67 & 148.05 & 138.79 & 37.35 & 43.56 & 3.58 & 3.63 \\
\hline 11 & MLTG (SB)- 14 & 28.67 & 100 & 6.67 & 18.33 & 1.78 & 19.33 & 27.73 & 15.91 & 11.98 & 75.33 & 151.28 & 138.34 & 43.68 & 41.4 & 3.82 & 3.53 \\
\hline 12 & MLTG (VB)- 1 & 32 & 120.67 & 4.33 & 10.33 & 1.75 & 12.33 & 13 & 11 & 6.46 & 56.01 & 140.36 & 127.82 & 41.92 & 43.18 & 3.72 & 3.74 \\
\hline 13 & MLTG (VB)- 2 & 31.67 & 121.67 & 6.67 & 16 & 1.86 & 20.67 & 17.73 & 21.75 & 16.92 & 83.25 & 156.55 & 123.18 & 37.87 & 44.61 & 3.48 & 3.57 \\
\hline 14 & MLTG (VB)- 5 & 33.67 & 123.67 & 5.33 & 13.67 & 1.82 & 18.33 & 13.2 & 17.4 & 12.06 & 69.69 & 141.79 & 123.53 & 43.92 & 48.32 & 3.86 & 3.79 \\
\hline 15 & MLTG (VB)- 6 & 30.67 & 118.67 & 7.33 & 20 & 1.87 & 24.33 & 20.1 & 15.2 & 10.53 & 67.95 & 179.37 & 157.38 & 37.51 & 40.13 & 3.48 & 3.38 \\
\hline 16 & MLTG (VB)- 7 & 32.33 & 119 & 6.33 & 14 & 1.8 & 16 & 26.93 & 13.87 & 10.59 & 78.58 & 156.2 & 147.6 & 41.62 & 47.25 & 3.66 & 3.66 \\
\hline 17 & MLTG (VB)- 8 & 29.67 & 119 & 6.67 & 20 & 1.72 & 20.33 & 30.17 & 14.7 & 10.46 & 75.11 & 151.55 & 143.52 & 39.4 & 43.39 & 3.42 & 3.6 \\
\hline 18 & MLTG (VB)- 9 & 31.67 & 119 & 6.33 & 12.33 & 2.29 & 20 & 11.5 & 13.1 & 10.73 & 87.58 & 147.59 & 128.15 & 43.23 & 45.41 & 3.87 & 3.87 \\
\hline 19 & MLTG (VB)- 11 & 33.67 & 119.67 & 7.67 & 20.33 & 1.91 & 22 & 18.83 & 23.5 & 16.33 & 69.7 & 146.19 & 150.78 & 42.45 & 43.81 & 3.88 & 3.88 \\
\hline 20 & MLTG(VB)- 12 & 30.67 & 118.67 & 5.67 & 17.33 & 2.31 & 22 & 17.33 & 15.61 & 14.23 & 85.24 & 139.89 & 121.86 & 41.97 & 42.91 & 3.83 & 3.83 \\
\hline 21 & INS-II-1 & 32.33 & 109 & 6 & 23.67 & 1.8 & 19 & 17.5 & 13.96 & 8.89 & 63.68 & 141.78 & 127.91 & 41.68 & 41.8 & 3.62 & 3.56 \\
\hline 22 & INS-II-3 & 29.67 & 106.67 & 5.33 & 21.67 & 1.81 & 23 & 11.5 & 17.8 & 13.59 & 74.1 & 131.26 & 130.13 & 40.66 & 41.08 & 3.66 & 3.48 \\
\hline 23 & INS-II-4 & 31.67 & 108.67 & 4.67 & 17 & 1.69 & 14.33 & 21.73 & 13.11 & 8.69 & 68.08 & 164.29 & 156.61 & 34.96 & 44.63 & 3.44 & 3.49 \\
\hline 24 & INS-II-5 & 30.67 & 110 & 5.33 & 14 & 1.7 & 13 & 8.33 & 12.14 & 8.7 & 71.71 & 152.04 & 145.2 & 43.16 & 39.65 & 3.87 & 3.58 \\
\hline 25 & INS-II-6 & 33.33 & 110.67 & 4 & 20.67 & 1.85 & 15.33 & 14.73 & 15.03 & 9.81 & 65.34 & 130.85 & 127.04 & 39.12 & 39.39 & 3.64 & 3.48 \\
\hline 26 & INS-II-7 & 31.33 & 109 & 5 & 19.33 & 1.76 & 14.67 & 12 & 14.87 & 10.58 & 69.48 & 134.08 & 119.7 & 45.46 & 40.82 & 3.81 & 3.57 \\
\hline
\end{tabular}




\begin{tabular}{|c|c|c|c|c|c|c|c|c|c|c|c|c|c|c|c|c|c|}
\hline 27 & INS-II-8 & 32 & 111 & 6 & 15.67 & 1.6 & 16.67 & 14.73 & 17.62 & 13.45 & 76.27 & 173.12 & 148.74 & 42.96 & 42.81 & 3.84 & 3.54 \\
\hline 28 & INS-II-9 & 33.67 & 111 & 5.33 & 20.33 & 1.72 & 11 & 21.43 & 12 & 7.12 & 57.31 & 150.48 & 145.73 & 40.31 & 42.01 & 3.71 & 3.55 \\
\hline 29 & INS-II-15 & 34 & 110 & 5.67 & 19 & 1.8 & 4.67 & 10.5 & 12.1 & 8.67 & 71.9 & 141.26 & 134.05 & 43.07 & 40.59 & 3.74 & 3.44 \\
\hline 30 & INS-II-24 & 32.67 & 108 & 4.33 & 16.33 & 1.81 & 17 & 14.57 & 13.67 & 10.68 & 77.93 & 134.1 & 123.51 & 39.91 & 41.31 & 3.56 & 3.42 \\
\hline 31 & TCGS 320 & 28.33 & 106 & 4.33 & 10 & 1.5 & 4.67 & 9 & 7.1 & 3.65 & 55.54 & 124.38 & 109.18 & 38.92 & 36.17 & 3.51 & 3.51 \\
\hline 32 & AVT-(D)-1397 & 31.67 & 116 & 4 & 16.67 & 2.63 & 14 & 13.73 & 12.18 & 9.18 & 74.72 & 157.2 & 129.41 & 42.46 & 45.96 & 3.57 & 3.51 \\
\hline 33 & AVT-(D)-1399 & 32.67 & 118.67 & 6 & 22 & 2.69 & 19.33 & 22.5 & 13.94 & 9.75 & 67.15 & 134.36 & 128.69 & 34.07 & 36.07 & 3.37 & 3.59 \\
\hline 34 & AVT-(D)-1407 & 33.67 & 119 & 4 & 15.67 & 1.67 & 18.33 & 19.23 & 13.7 & 8.89 & 70.83 & 138.47 & 150.41 & 36.82 & 44.86 & 3.44 & 3.55 \\
\hline 35 & AVT-(D)-1416 & 30 & 109 & 4 & 18.33 & 1.91 & 19.33 & 15.17 & 14.41 & 10.24 & 69.51 & 143.46 & 163.6 & 41.61 & 41.82 & 3.84 & 3.48 \\
\hline 36 & AVT-(D)-1425 & 31.33 & 109.67 & 4.67 & 30.67 & 1.79 & 22 & 18.5 & 13.62 & 10.44 & 75.05 & 144.85 & 146.11 & 46.93 & 42.24 & 3.79 & 3.42 \\
\hline 37 & AVT-(D)-1426 & 32 & 117.33 & 4.33 & 14.67 & 1.73 & 17.33 & 27.17 & 14.34 & 10.49 & 72.68 & 148.65 & 123.56 & 37.01 & 38.51 & 3.48 & 3.51 \\
\hline 38 & AVT-(D)-1429 & 33.67 & 120.67 & 4.67 & 17.33 & 1.71 & 16.67 & 15.43 & 12.2 & 8.15 & 66.85 & 122.09 & 120.74 & 34.45 & 43.44 & 3.44 & 3.55 \\
\hline 39 & AVT-(D)-1433 & 32.67 & 117.67 & 4.33 & 12.33 & 1.89 & 16 & 16.5 & 13.02 & 8.04 & 60.44 & 147.39 & 125.76 & 35.11 & 38.71 & 3.61 & 3.48 \\
\hline 40 & AVT-(D)-1437 & 31.67 & 117.33 & 4.33 & 17 & 1.81 & 13.33 & 12.5 & 12.16 & 8.15 & 64.32 & 156.82 & 129.84 & 38.87 & 40.3 & 3.64 & 3.47 \\
\hline 41 & K-6 & 26.33 & 102.33 & 4.67 & 16.67 & 1.87 & 22.67 & 12.4 & 13.1 & 8.57 & 65.34 & 255.34 & 134.52 & 36.24 & 38.16 & 3.66 & 3.48 \\
\hline 42 & ABHAYA & 29.67 & 105.33 & 4.67 & 17 & 2.91 & 23.67 & 10.23 & 12.4 & 8.6 & 69.37 & 151.77 & 132.43 & 39.54 & 41.94 & 3.6 & 3.51 \\
\hline 43 & DHARANI & 29.33 & 103.33 & 4 & 17 & 1.75 & 21.67 & 15 & 15.83 & 10.86 & 69.78 & 146.16 & 133.25 & 38.44 & 41.46 & 3.64 & 3.42 \\
\hline 44 & NARAYANI & 29 & 108.67 & 5 & 12.67 & 1.68 & 18 & 22.67 & 17.2 & 10.89 & 63.28 & 180.05 & 168.6 & 36.92 & 36.74 & 3.53 & 3.48 \\
\hline 45 & IET 1509 & 32.67 & 109.67 & 3 & 14.67 & 1.86 & 22 & 18.33 & 13.9 & 9.75 & 70 & 132.57 & 137.6 & 35.59 & 41.91 & 3.66 & 3.38 \\
\hline 46. & IET 1513 & 34.67 & 115.67 & 4 & 18 & 1.89 & 16 & 21.17 & 12.62 & 7.99 & 62.05 & 157.33 & 148.85 & 39.17 & 36.85 & 3.47 & 3.49 \\
\hline 47 & IET 1524 & 35.67 & 117 & 3.67 & 18.33 & 1.86 & 24.67 & 32.17 & 15.07 & 9.93 & 64.9 & 148.54 & 149.65 & 35.16 & 40.71 & 3.71 & 3.49 \\
\hline 48 & IET 1530 & 37.67 & 117.67 & 4 & 16.33 & 1.59 & 16.33 & 22.43 & 18.33 & 12.6 & 71 & 142.23 & 144.69 & 40.53 & 43.74 & 3.48 & 3.34 \\
\hline 49 & IET 1531 & 35 & 116.33 & 3.67 & 13.67 & 1.85 & 26.67 & 19.73 & 17.4 & 12.2 & 71.29 & 162.95 & 147.91 & 35.09 & 38.31 & 3.67 & 3.49 \\
\hline 50 & IET 1532 & 31.67 & 108 & 4 & 17 & 1.75 & 17.67 & 21.67 & 18.5 & 10.97 & 63.9 & 149.43 & 148.27 & 39.63 & 38.11 & 3.71 & 3.51 \\
\hline & General Mean & 30.92 & 109.85 & 5.18 & 17.25 & 1.86 & 18.03 & 18.13 & 15.18 & 10.19 & 67.66 & 151.26 & 138.79 & 39.86 & 41.42 & 3.7 & 3.7 \\
\hline & C.V. & 1.57 & 0.72 & 9.6 & 13.33 & 3.85 & 9.72 & 10.46 & 7.64 & 7.21 & 5.1 & 2.24 & 1.03 & 4.69 & 3.84 & 1.68 & 0.98 \\
\hline
\end{tabular}


However, selection based on some identified traits regardless of interactions among them and with grain yield components may mislead the plant breeders to accomplish their main breeding purposes (Garcia del Moral et al., 2003).

Clustering based on studied traits separated the groundnut genotypes into five main groups under rainfed condition (Figure2). There were MLTG(SB)-1, MLTG(SB)-2, Narayani, MLTG(SB)-4, MLTG(SB)-6, MLTG(SB)-13, INS-II-4, INS-II-9, IET 1531, IET 1532, MLTG(VB)-1, TCGS-320, MLTG(VB)-2, MLTG(VB)-8, MLTG(VB)-9, MLTG(VB)-12, INS-II-24, INS-II-7, MLTG(VB)-11， AVT(D)-1407， IET1509, IET 1530, INS-II-16, INS-II-1, AVT(D)1397, AVT(D)-1433,AVT(D)-1437, AVT(D)1399, MLTG(SB)-10, Abhaya, Dharani, INSII-8, IET 1531, INS-II-5, MLTG(VB)-6, MLTG(SB)-14, INS-II-15, INS-II-3, MLTG(VB)-5, MLTG(SB)-5, IET-1524, MLTG(VB)-7, AVT(D)-1426, AVT(D)-1425, AVT(D)-1416 and AVT(D)-1429 in one group and genotype MLTG(SB)-3 in second cluster, the genotype MLTG(SB)-12 in third cluster, MLTG(SB)-11 in fourth cluster and likewise, K-6 in fifth cluster.

Therefore, it seems that breeding for pod yield under rainfed conditions genotypes MLTG(SB)-3 from second cluster, MLTG(SB)-6, MLTG(VB)-11 and MLTG(VB)-2 from first cluster could be utilized. Mean performance of fifty groundnut genotypes under rainfed conditions were presented in table 1 . This suggested that the observed differences in groundnut genotypes were sufficient to provide some facilities for selecting the most favorable genotypes to improve pod yield performance.

For a trait to be considered as a selection criterion in grain yield improvement program it must be associated with grain yield and it is therefore, essential to determine whether grain yield was associated with a particular trait. We found that number of pods per plant, dry matter per plant, SLA at 80 DAS, number of sound mature kernels per plant, kernel yield per plant and SLA at 60 DAS were the most related traits with pod yield per plant. Cluster analysis results proved that the abovementioned traits were the variables most closely related to pod yield as well as drought tolerance.

These results suggest that selections should be based on the number of pods per plant, dry matter per plant, SLA at 80 DAS, number of sound mature kernels per plant, kernel yield per plant and SLA at 60 DAS developing new groundnut genotypes with drought tolerance traits.

Clustering based on groundnut genotypes separated the measured traits into three main groups under rainfed conditions. Genotypes are distributed among all cluster groups, which implied that genetically different genotypes were identified with pod yield performance.

It is reasonable to assume that the genetic basis of pod yield per plant and other measured traits in these genotypes is different, which would enable groundnut breeders to combine these different sources of genetic variability to improve pod yield per plant as well as other measured traits in their breeding programs. Maximum genetic variation is expected from crosses that involve parents from clusters characterized by maximum distance. Crosses between genotypes selected on the basis of special merits are, therefore, expected to provide relatively better genetic recombination in their progenies. Hence, it seems that for improving pod yield per plant under rainfed conditions, genotypes MLTG(SB)-3, MLTG(SB)-6, MLTG(VB)-11 and MLTG(VB)-2 are good candidates. 


\section{References}

Basnet, K. M., Adhikari, N. R. and Pandey, M. P. 2014. Multivariate analysis among Nepalese and exotic mungbean (Vigna radiata $\mathrm{L}$. Wilczek) genotypes based on the qualitative parameters. Universal J. Agricultural Research. 2(5): 147-155.

Binod Kumar, Anil Pandey and Sanjay, K. R. Singh. 2013. Multivariate analysis of genetic divergence among Indian Mustard (Brassica juncea L. Czern \& Coss) genotypes in relation to oil quality traits. The Bioscan. 8(4): 15451549.

Garcia del Moral, L. F., Rharrabti, Y., Villegas, D. and Royo, C. 2003. Evaluation of grain yield and its components in durum wheat under
Mediterranean conditions: an ontogenic approach. Agronomy J. 95:266-274.

Johnson, H. W., Robinson, H. F. and Cromstock, R. E. 1956. Estimation of genetic and environmental variability in soybeans. Agronomy J. 47: 314-318

Katiyar, P. K., Dixit, G. P., Singh, B. B., Ali Hasmat and Dubey, M.K. 2009. Nonhierarchical Euclidean cluster analysis for genetic divergence in mungbean cultivars. J. Food Lugumes. 22(1): 3436.

Singh Rashmi, Ali Hasmat and Pathak Varun. 2010. Non-Hierarchical Euclidean cluster analysis in mungbean. Trends in Biosciences. 3(2): 135-136.

Yogendra Prasad, Kamleshwar Kumar and Mishra, S. B. 2013. Role of genetic diversity for exploiting the heterosis in pigeaonpea (Cajanus cajan (L.) Mill sp.). The Bioscan. 8(2): 409-416.

\section{How to cite this article:}

Kavitha, G. and Reddi Sekhar, M. 2018. Phenotypic Divergence for Yield and Drought Tolerance Related Traits in Groundnut Genotypes under Rainfed Conditions. Int.J.Curr.Microbiol.App.Sci. 7(03): 3000-3006. doi: https://doi.org/10.20546/ijcmas.2018.703.347 